Article

\title{
On the Causality and K-Causality between Measures
}

\author{
Tomasz Miller ${ }^{1,2}$ \\ 1 Faculty of Mathematics and Information Science, Warsaw University of Technology, ul. Koszykowa 75, \\ 00-662 Warsaw, Poland; T.Miller@mini.pw.edu.pl \\ 2 Copernicus Center for Interdisciplinary Studies, ul. Szczepańska 1/5, 31-011 Kraków, Poland \\ Academic Editors: Mariusz P. Dąbrowski, Manuel Krämer and Vincenzo Salzano \\ Received: 31 January 2017; Accepted: 11 March 2017; Published: 20 March 2017
}

\begin{abstract}
Drawing from the optimal transport theory adapted to the Lorentzian setting, we propose and study the extension of the Sorkin-Woolgar causal relation $\mathrm{K}^{+}$onto the space of Borel probability measures on a given spacetime. We show that it retains its fundamental properties of transitivity and closedness. Furthermore, we list and prove several characterizations of this relation, including the "measure-theoretic" analogue of the characterization of $\mathrm{K}^{+}$in terms of time functions.
\end{abstract}

Keywords: causal relations; Lorentzian optimal transport theory; time functions

\section{Introduction}

One of the vital fibers of mathematical relativity is causality theory; i.e., the study of the causal properties of spacetimes. The fundamental role in this theory is played by certain binary relations on a given spacetime, designed to describe which events (e.g., points of spacetime) are causally connected, and which can never constitute links of a cause-effect chain. The two most important such relations_called $I^{+}$and $J^{+}$-involve pairs of events $(p, q)$ which can be connected by means of a future-directed chronological curve (which is denoted $p \ll q$ ) or a future-directed causal curve (which is often denoted $p \preceq q$ ), respectively. For a concise review of causality theory, the reader is referred to [1] (see also [2-6] for the full exposition).

Motivated by the approach to Lorentzian noncommutative geometry developed in [7-11]—where spacetime usually can no longer be regarded as composed of point-like events but nevertheless possesses a causal structure-we have recently proposed and studied a natural extension of the relation $\preceq$ onto the space $\mathscr{P}(\mathcal{M})$ of Borel probability measures on a given spacetime $\mathcal{M}$ [12], which relies on the notion of a coupling drawn from the optimal transport theory [13,14]. Given a pair of measures $\mu, v \in \mathscr{P}(\mathcal{M})$, we call $\omega \in \mathscr{P}\left(\mathcal{M}^{2}\right)$ a coupling of $\mu$ and $\nu$ iff the latter two measures are $\omega^{\prime}$ s marginals; that is, iff $\pi_{\sharp}^{1} \omega=\mu$ and $\pi_{\sharp}^{2} \omega=v$, where $\pi^{i}: \mathcal{M}^{2} \rightarrow \mathcal{M}, i=1,2$ denote the canonical projections. With $\Pi(\mu, v)$ denoting the set of all such couplings, we have put forward the following definition.

Definition 1. Let $\mathcal{M}$ be a spacetime. For any $\mu, v \in \mathscr{P}(\mathcal{M})$, we say that $\mu$ causally precedes $v$ (symbolically $\mu \preceq v)$ iff there exists a causal coupling of $\mu$ and $\nu$, by which we mean any $\omega \in \Pi(\mu, v)$ such that $\omega\left(J^{+}\right)=1$.

Let us emphasize that the quantity $\omega\left(J^{+}\right)$is well-defined, because $J^{+}$is a Borel subset of $\mathcal{M}^{2}$ ([12], Section 3). The name "causal coupling" was independently coined in [15].

This definition rigorously encapsulates the following common intuition: infinitesimal portions of probability distributions can propagate in spacetime only along future-directed causal curves. The thus-obtained formalism can be successfully used to model the causal time-evolution of spatially distributed physical quantities [16] and wave packets [17], and to quantify the breakdown of causality in various physical models. Although the idea of studying causality for spread objects is by no means new (e.g., [18-25]), the tools of the modern optimal transport theory adapted to the Lorentzian setting 
allow us to cast some new light on the previous approaches and significantly extend them. See [17] for concrete physical applications and a more detailed discussion.

Even though $I^{+}, J^{+}$play the central role in mathematical relativity, these relations are not the only ones studied in causality theory, with the so-called Sorkin-Woolgar relation $\mathrm{K}^{+} \supseteq \mathrm{J}^{+}$providing an important example. It is defined in [26] as the smallest transitive and topologically closed relation containing $I^{+}$, and it was originally studied in the context of reformulating causality theory along topological and order-theoretic lines, which in particular would accommodate for low-regularity metrics. It turns out that $K^{+}$is additionally antisymmetric (and hence a partial order) precisely when the spacetime $\mathcal{M}$ is stably causal [27], in which case it possesses the following characterization in terms of time functions (i.e., continuous real-valued functions on $\mathcal{M}$ which are strictly increasing along any future-directed causal curve) [28].

Theorem 1 (Minguzzi). Let $\mathcal{M}$ be a stably causal spacetime and $p, q \in \mathcal{M}$. Then,

$$
(p, q) \in K^{+} \quad \Leftrightarrow \quad \text { For every time function } \mathcal{T} \quad \mathcal{T}(p) \leq \mathcal{T}(q) .
$$

In his work [27], E. Minguzzi calls $K^{+}$"one of the most important [relations] for the development of causality theory". It is thus natural to ask whether the formalism developed in [12] for $\mathrm{J}^{+}$can be adapted to $\mathrm{K}^{+}$. Indeed, Definition 1 can be immediately modified to extend the relation $\mathrm{K}^{+}$(instead of $\mathrm{J}^{+}$) onto $\mathscr{P}(\mathcal{M})$ (see Definition 2 below). The aim of this paper is to study the thus-obtained relation between measures. More concretely, in Section 2 we show that the Sorkin-Woolgar relation-when extended onto $\mathscr{P}(\mathcal{M})$-retains its fundamental properties of transitivity and closedness (Proposition 1). Then, we establish its various characterizations (Theorem 2), in particular providing the "measure-theoretic" counterpart of equivalence (1). All of that generalizes and sheds new light on some of the results presented in [12], which is briefly discussed in Section 3. Finally, the Appendix A outlines the definitions and theorems from the probability theory on Polish spaces used in the article.

\section{Results}

From now on, the term "measure" will always stand for "Borel probability measure".

Analogously as for the relations $I^{+}$and $J^{+}$, for any $\mathcal{X} \subseteq \mathcal{M}$, one introduces the notation $K^{+}(\mathcal{X}):=\pi^{2}\left((\mathcal{X} \times \mathcal{M}) \cap K^{+}\right)$and $K^{-}(\mathcal{X}):=\pi^{1}\left((\mathcal{M} \times \mathcal{X}) \cap K^{+}\right)$. By definition, $K^{+}$is closed, and hence for any compact $C \subseteq \mathcal{M}$, the sets $K^{ \pm}(C)$ are closed. More generally, however, for $\mathcal{X} \subseteq \mathcal{M}$ which is only Borel, the sets $K^{ \pm}(\mathcal{X})$ need not be Borel. Nevertheless, being projections of Borel sets, they are universally measurable, which means that for any measure $\mu \in \mathscr{P}(\mathcal{M})$, the sets $K^{ \pm}(\mathcal{X})$ are Borel up to a $\mu$-negligible set, and therefore the quantity $\mu\left(K^{ \pm}(\mathcal{X})\right)$ is well-defined [29].

As announced in the Introduction, let us put forward the following definition of the Sorkin-Woolgar relation between measures.

Definition 2. Let $\mathcal{M}$ be a spacetime. For any $\mu, v \in \mathscr{P}(\mathcal{M})$, we say that $\mu \mathrm{K}$-causally precedes $v$ (symbolically $\mu \preceq_{K} v$ ) iff there exists a K-causal coupling of $\mu$ and $\nu$, by which we mean any $\omega \in \Pi(\mu, v)$ such that $\omega\left(K^{+}\right)=1$.

As a first result, let us observe that the defining properties of the Sorkin-Woolgar relation—closedness and transitivity—still hold after extending it onto $\mathscr{P}(\mathcal{M})$.

Proposition 1. The relation $\preceq_{K}$ on $\mathscr{P}(\mathcal{M})$ is reflexive and transitive, as well as closed, by which we mean that the set $\left\{(\mu, v) \in \mathscr{P}(\mathcal{M})^{2} \mid \mu \preceq_{K} v\right\}$ is closed in $\mathscr{P}(\mathcal{M})^{2}$ endowed with the (product) narrow topology (for the definition of the narrow topology, see Appendix A). 
Proof. Reflexivity and transitivity can be shown exactly as in the proof of ([12], Theorem 11) with $\preceq$ and $J^{+}$replaced by $\preceq_{K}$ and $K^{+}$.

As for the closedness, take any sequences $\left(\mu_{n}\right),\left(v_{n}\right) \subseteq \mathscr{P}(\mathcal{M})$ satisfying $\mu_{n} \preceq_{K} v_{n}$ for all $n \in \mathbb{N}$, and suppose that they converge, respectively, to some $\mu, v \in \mathscr{P}(\mathcal{M})$ in the narrow topology; i.e., that

$$
\forall f \in C_{b}(\mathcal{M}) \int_{\mathcal{M}} f d \mu_{n} \rightarrow \int_{\mathcal{M}} f d \mu \text { and } \int_{\mathcal{M}} f d v_{n} \rightarrow \int_{\mathcal{M}} f d v .
$$

What we need to prove is that $\mu \preceq_{K} v$. To this end, let $\omega_{n}$ be the $K$-causal coupling of $\mu_{n}$ and $v_{n}$ for every $n \in \mathbb{N}$. We claim that the sequence $\left(\omega_{n}\right) \subseteq \mathscr{P}\left(\mathcal{M}^{2}\right)$ has a narrowly convergent subsequence. Indeed, the sets $\left\{\mu_{n}\right\}_{n \in \mathbb{N}},\left\{v_{n}\right\}_{n \in \mathbb{N}}$ are relatively compact (in the narrow topology) and hence tight by the Prokhorov theorem (see Appendix A). However, this implies that the set $\bigcup_{i, j \in \mathbb{N}} \Pi\left(\mu_{i}, v_{j}\right)$ is also tight ([14], Lemma 4.4) and hence relatively compact. Since $\left(\omega_{n}\right)$ is contained in $\bigcup_{i, j \in \mathbb{N}} \Pi\left(\mu_{i}, v_{j}\right)$, it possesses a subsequence (which we keep denoting as $\left(\omega_{n}\right)$ ) convergent to some $\omega \in \mathscr{P}\left(\mathcal{M}^{2}\right)$.

It now remains to show that $\omega$ is a $K$-causal coupling of $\mu$ and $v$. In order to prove that $\omega \in \Pi(\mu, v)$, observe first that for any $g \in C_{b}(\mathcal{M})$

$$
\int_{\mathcal{M}} g d\left(\pi_{\sharp}^{1} \omega\right)=\int_{\mathcal{M}}\left(g \circ \pi^{1}\right) d \omega=\lim _{n \rightarrow+\infty} \int_{\mathcal{M}}\left(g \circ \pi^{1}\right) d \omega_{n}=\lim _{n \rightarrow+\infty} \int_{\mathcal{M}} g d \mu_{n}=\int_{\mathcal{M}} g d \mu,
$$

which proves that $\pi_{\sharp}^{1} \omega=\mu$. The equality $\pi_{\sharp}^{2} \omega=v$ is proven analogously. Finally, in order to show that $\omega\left(K^{+}\right)=1$, invoke the Portmanteau theorem (see Appendix A), which toghether with the closedness of $K^{+}$yields $\omega\left(K^{+}\right) \geq \limsup _{n \rightarrow+\infty} \omega_{n}\left(K^{+}\right)=1$.

We now provide several characterizations of the relation $\preceq_{K}$ for measures, which constitutes the main result of the paper.

Theorem 2. Let $\mathcal{M}$ be a spacetime. For any $\mu, v \in \mathscr{P}(\mathcal{M})$, consider the following list of conditions.

$1 \bullet \quad \mu \preceq_{K} v$.

$2^{\bullet}$ For any compact subset $C \subseteq \mathcal{M}$,

$$
\mu\left(K^{+}(C)\right) \leq v\left(K^{+}(C)\right)
$$

3- For any Borel subset $\mathcal{X} \subseteq \mathcal{M}$ such that $\mathrm{K}^{+}(\mathcal{X}) \subseteq \mathcal{X}$,

$$
\mu(\mathcal{X}) \leq v(\mathcal{X})
$$

4. For any time function $\mathcal{T}$ and any $\alpha \in \mathbb{R}$,

$$
\mu\left(\mathcal{T}^{-1}((\alpha,+\infty))\right) \leq v\left(\mathcal{T}^{-1}((\alpha,+\infty))\right)
$$

5- For any bounded time function $\mathcal{T}$,

$$
\int_{\mathcal{M}} \mathcal{T} d \mu \leq \int_{\mathcal{M}} \mathcal{T} d v
$$

Then, we have the following equivalences and implications $1^{\bullet} \Leftrightarrow 2^{\bullet} \Leftrightarrow 3^{\bullet} \Rightarrow 4^{\bullet} \Rightarrow 5^{\bullet}$. Moreover, if $\mathcal{M}$ is causally continuous, then all above conditions are equivalent.

Observe that conditions $4^{\bullet}$ and $5^{\bullet}$ implicitly assume that $\mathcal{M}$ is stably causal. Condition $5^{\bullet}$ is a natural extension of Minguzzi's condition (1) onto measures. Notice, however, that we assume here the causal continuity of $\mathcal{M}$ for the equivalence $1^{\bullet} \Leftrightarrow 5^{\bullet}$ to hold. 
All above conditions (with the exception of $4^{\bullet}$ ) are analogues of those studied in [12] in the context of the relation $\mathrm{J}^{+}$, and in fact they reduce to them for the case of causally simple spacetimes. However, the properties of $\mathrm{K}^{+}$allow these analogues to work in a broader class of spacetimes. Some parts of the following proof simply mimic the argumentation presented in [12], while others are significantly different, thus offering an alternative way to reach the results of the cited work. In particular, the crucial role will be played by the following fact, adapted from a more general result obtained by Suhr, cf. ([15], Theorem 2.5).

Theorem 3 (Suhr). For any spacetime $\mathcal{M}$ and any $\mu, v \in \mathscr{P}(\mathcal{M})$, the following conditions are equivalent

(i) $\mu \preceq_{K} v$.

(ii) For any Borel $\mathcal{B} \subseteq \mathcal{M}$,

$$
\mu(\mathcal{B}) \leq v\left(K^{+}(\mathcal{B})\right) \quad \text { and } \quad \mu\left(K^{-}(\mathcal{B})\right) \geq v(\mathcal{B}) .
$$

We will also need the following lemma, the proof of which is a straightforward adaptation of that of ([12], Proposition 1).

Lemma 1. For any spacetime $\mathcal{M}$ and any $\mathcal{X} \subseteq \mathcal{M}$, denote $\mathcal{X}^{c}:=\mathcal{M} \backslash \mathcal{X}$. We have the equivalence of inclusions $K^{+}(\mathcal{X}) \subseteq \mathcal{X} \Leftrightarrow K^{-}\left(\mathcal{X}^{c}\right) \subseteq \mathcal{X}^{c}$.

Proof of Theorem 2. $1^{\bullet} \Rightarrow 2^{\bullet}$ By the closedness of $K^{+}$, the set $K^{+}(C)$ is closed, and hence Borel, for any compact $C$. Denoting the characteristic function of $K^{+}(C)$ by $\chi$, the inequality $\chi(p) \leq \chi(q)$ holds for all $(p, q) \in K^{+}$by the transitivity of $K^{+}$. By assumption, there exists a $K$-causal coupling $\omega \in \Pi(\mu, v)$, and one has that

$$
\begin{aligned}
\mu\left(K^{+}(C)\right) & =\int_{\mathcal{M}} \chi(p) d \mu(p)=\int_{\mathcal{M}^{2}} \chi(p) d \omega(p, q)=\int_{K^{+}} \chi(p) d \omega(p, q) \leq \int_{K^{+}} \chi(q) d \omega(p, q) \\
& =\int_{\mathcal{M}^{2}} \chi(q) d \omega(p, q)=\int_{\mathcal{M}} \chi(q) d v(q)=v\left(K^{+}(C)\right) .
\end{aligned}
$$

$2^{\bullet} \Rightarrow 3^{\bullet}$ Let the set $\mathcal{X}$ be as specified in $3^{\bullet}$, and let $C$ be any compact subset of $\mathcal{X}$. Then also $K^{+}(C) \subseteq K^{+}(\mathcal{X}) \subseteq \mathcal{X}$, and one has that

$$
\mu(C) \leq \mu\left(K^{+}(C)\right) \leq v\left(K^{+}(C)\right) \leq v(\mathcal{X}),
$$

where the inequality holds by $2^{\bullet}$. Every Borel probability measure on a Polish space is inner regular (see Appendix A); therefore, by taking the supremum over all compacts $C \subseteq \mathcal{X}$, we obtain that

$$
\mu(\mathcal{X})=\sup \{\mu(C) \mid C \subseteq \mathcal{X} \text { compact }\} \leq v(\mathcal{X}) .
$$

$3^{\bullet} \Rightarrow 1^{\bullet}$ It suffices to prove that condition ii) in Theorem 3 is satisfied.

To this end, observe that the first inequality in ii) follows directly from $3^{\bullet}$ with $\mathcal{X}:=K^{+}(\mathcal{B})$ (up to a $\mu$-negligible set), and the obvious inequality $\mu(\mathcal{B}) \leq \mu\left(K^{+}(\mathcal{B})\right.$ ). In order to obtain the second

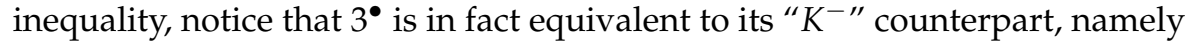

$3^{\prime \bullet}$ For any Borel subset $\mathcal{Y} \subseteq \mathcal{M}$ such that $K^{-}(\mathcal{Y}) \subseteq \mathcal{Y}$,

$$
\mu(\mathcal{Y}) \geq v(\mathcal{Y}) .
$$

Indeed, to move between conditions $3^{\bullet}$ and $3^{\prime \bullet}$, simply take $\mathcal{Y}=\mathcal{X}^{c}$ and invoke Lemma 1. 
The second inequality in condition ii) of Theorem 3 follows from $3^{\prime \bullet}$ with $\mathcal{Y}:=K^{-}(\mathcal{B})$ (up to a $v$-negligible set) and the obvious inequality $v\left(K^{-}(\mathcal{B})\right) \geq v(\mathcal{B})$.

$3^{\bullet} \Rightarrow 4^{\bullet}$ Take any time function $\mathcal{T}$ (from now on, we implicitly assume that $\mathcal{M}$ is stably causal) and any $\alpha \in \mathbb{R}$. On the strength of $3^{\bullet}$, we only have to show that $K^{+}\left(\mathcal{T}^{-1}((\alpha,+\infty))\right) \subseteq \mathcal{T}^{-1}((\alpha,+\infty))$.

To this end, let $q \in \mathcal{M}$ be such that there exists $p \in \mathcal{T}^{-1}((\alpha,+\infty))$ with $(p, q) \in K^{+}$. By Theorem 1, we immediately obtain that $\mathcal{T}(q) \geq \mathcal{T}(p)>\alpha$, and so $q \in \mathcal{T}^{-1}((\alpha,+\infty))$ as well.

$4^{\bullet} \Rightarrow 5^{\bullet}$ Fix a bounded time function $\mathcal{T}$. Reasoning similarly as in the proof of ([12], Theorem 7 , $3^{\bullet} \Rightarrow 2^{\bullet}$ ), denote $m:=\inf \mathcal{T}$ and $M:=\sup \mathcal{T}$ and for every $n \in \mathbb{N}$ define the simple function

$$
s_{n}:=m+\sum_{k=1}^{n-1} \frac{M-m}{n} \chi_{\mathcal{F}_{k}^{(n)}}
$$

where $\mathcal{F}_{k}^{(n)}:=\mathcal{T}^{-1}\left(\left(m+k \frac{M-n}{n},+\infty\right)\right), k=1,2, \ldots, n-1$, and the functions $\chi_{\mathcal{F}_{k}^{(n)}}$ denote their respective characteristic functions. On the strength of $4^{\bullet}$, we have that

$$
\int_{\mathcal{M}} s_{n} d \mu=m+\sum_{k=1}^{n-1} \frac{M-m}{n} \mu\left(\mathcal{F}_{k}^{(n)}\right) \leq m+\sum_{k=1}^{n-1} \frac{M-m}{n} v\left(\mathcal{F}_{k}^{(n)}\right)=\int_{\mathcal{M}} s_{n} d v .
$$

The functions $s_{n}$ are designed so as to satisfy $0<\mathcal{T}-s_{n} \leq \frac{M-m}{n}$ for all $n \in \mathbb{N}$. The somewhat technical justification (which we skip here) can be found in the above-mentioned proof in [12]. This property allows us to go with $n$ to infinity in (7) by Lebesgue's dominant convergence theorem, which yields (5).

$5^{\bullet} \Rightarrow 2^{\bullet}$ Fix a compact set $C$ and define $\mathcal{Y}:=K^{+}(C)^{c}$. By the closedness of $K^{+}(C)$ and Lemma 1 , $\mathcal{Y}$ is an open set with the property that $K^{-}(\mathcal{Y}) \subseteq \mathcal{Y}$.

We go along the lines of the suitably modified proof of ([12], Theorem $7,2^{\bullet} \Rightarrow 3^{\bullet}$ ). To begin with, let $\eta \in \mathscr{P}(\mathcal{M})$ be an admissible measure, which by definition satisfies $\eta(U)>0$ for all open $U \subseteq \mathcal{M}$ and $\eta\left(\partial I^{ \pm}(p)\right)=0$ for all $p \in \mathcal{M}$ ([2], Definition 3.19). For any $\lambda \in(0,1]$, the measure $\eta_{\lambda}:=\lambda \eta+(1-\lambda) \eta(. \cap \mathcal{Y})$ is another admissible measure. Its associated future volume function $t_{\lambda}^{+}$, defined for any $p \in \mathcal{M}$ via

$$
\begin{aligned}
t_{\lambda}^{+}(p) & :=-\eta_{\lambda}\left(I^{+}(p)\right)=-\lambda \eta\left(I^{+}(p)\right)-(1-\lambda) \eta\left(I^{+}(p) \cap \mathcal{Y}\right) \\
& =-\eta\left(I^{+}(p) \cap \mathcal{Y}\right)-\lambda\left(\eta\left(I^{+}(p)\right)-\eta\left(I^{+}(p) \cap \mathcal{Y}\right)\right)=-\eta\left(I^{+}(p) \cap \mathcal{Y}\right)-\lambda \eta\left(I^{+}(p) \backslash \mathcal{Y}\right)
\end{aligned}
$$

is a (bounded) time function provided $\mathcal{M}$ is causally continuous (this is where this assumption comes into play). Take now any sequence of strictly increasing functions $\left(\varphi_{n}\right) \subseteq C_{b}(\mathbb{R})$ pointwise convergent to the negative characteristic function of the interval $(-\infty, 0)$, denoted here as $-\chi_{<0}$ (for instance, one can take $\left.\varphi_{n}(x):=-\frac{1}{2}+\frac{1}{2} \tanh \left(n^{2} x+n\right), n \in \mathbb{N}\right)$. By $5^{\bullet}$, for all $\lambda \in(0,1]$ and all $n \in \mathbb{N}$, it is true that

$$
\int_{\mathcal{M}} \varphi_{n}\left(t_{\lambda}^{+}(p)\right) d \mu(p) \leq \int_{\mathcal{M}} \varphi_{n}\left(t_{\lambda}^{+}(p)\right) d v(p)
$$

Using the dominant convergence theorem twice, first for taking $\lambda \rightarrow 0^{+}$, and then $n \rightarrow+\infty$, we obtain that

$$
-\int_{\mathcal{M}} \chi_{<0}\left(-\eta\left(I^{+}(p) \cap \mathcal{Y}\right)\right) d \mu(p) \leq-\int_{\mathcal{M}} \chi_{<0}\left(-\eta\left(I^{+}(p) \cap \mathcal{Y}\right)\right) d v(p) .
$$


It is now crucial to observe that the map $p \mapsto \eta\left(I^{+}(p) \cap \mathcal{Y}\right)$ is positive on $\mathcal{Y}$ and zero on $\mathcal{Y}^{c}$. Indeed, for $p \in \mathcal{Y}$, the set $I^{+}(p) \cap \mathcal{Y}$ is open and nonempty by the openness of $\mathcal{Y}$, and therefore $\eta\left(I^{+}(p) \cap \mathcal{Y}\right)>0$ by the very definition of an admissible measure. Conversely, if $\eta\left(I^{+}(p) \cap \mathcal{Y}\right)>0$, then there exists $q \in \mathcal{Y}$ such that $p \ll q$, which in turn means that $p \in I^{-}(q) \subseteq K^{-}(q) \subseteq K^{-}(\mathcal{Y}) \subseteq \mathcal{Y}$.

By the above observation, the integrands in (8) are nothing but the characteristic function of $\mathcal{Y}$, and hence (8) boils down to $-\mu(\mathcal{Y}) \leq-v(\mathcal{Y})$, which in turn yields $2^{\bullet}$, because

$$
\mu\left(K^{+}(C)\right)=\mu\left(\mathcal{Y}^{c}\right)=1-\mu(\mathcal{Y}) \leq 1-v(\mathcal{Y})=v\left(\mathcal{Y}^{c}\right)=v\left(K^{+}(C)\right) .
$$

Remark 1. If one replaces in condition $5^{\bullet}$ the term "bounded" with " $\mu$-and $v$-integrable", and/or the term "time" with "temporal", "smooth time", "smooth causal", or "continuous causal", the thus-obtained condition is equivalent with $5^{\bullet}$.

Proof. To begin with, let $\tau$ stand for any term from the set temporal, smooth time, time, smooth causal, continuous causal $\}$. Let us assume that inequality (5) holds for all bounded $\tau$ functions and take any $\mu$ - and $\nu$-integrable $\tau$ function $\mathcal{T}$. For every $n \in \mathbb{N}$, consider the bounded function $n \tanh \left(\frac{1}{n} \mathcal{T}\right)$, which is also $\tau$. By assumption,

$$
\int_{\mathcal{M}} n \tanh \left(\frac{1}{n} \mathcal{T}(p)\right) d \mu(p) \leq \int_{\mathcal{M}} n \tanh \left(\frac{1}{n} \mathcal{T}(p)\right) d v(p) .
$$

Notice now that $\left|n \tanh \left(\frac{1}{n} \mathcal{T}\right)\right| \leq \mathcal{T}$ and that $n \tanh \left(\frac{1}{n} \mathcal{T}\right) \rightarrow \mathcal{T}$ pointwise, and hence, by the dominant convergence theorem, inequality (9) becomes (5) as $n$ approaches infinity.

The converse implication holds trivially, because every bounded $\tau$ function is $\mu$-integrable for any $\mu \in \mathscr{P}(\mathcal{M})$.

We now move to proving that replacing "time" in $5^{\bullet}$ with any term from the set temporal, smooth time, time, smooth causal, continuous causal $\}$ yields a condition equivalent to $5^{\bullet}$. Notice that the "continuous causal" version of $5^{\bullet}$ implies all other versions, whereas the "temporal" version of $5^{\bullet}$ is implied by every other version. We thus only have to show that the "temporal" version implies the "continuous causal" one.

To this end, assume that $f \in C_{b}(\mathcal{M})$ is a causal function and let $t$ be a fixed bounded time function. Then, for any $n \in \mathbb{N} f+\frac{1}{n} t$ is another bounded time function, which in turn can be approximated by a sequence of temporal functions $\left(\mathcal{T}_{n, m}\right)_{m \in \mathbb{N}}$ such that $\left|f+\frac{1}{n} t-\mathcal{T}_{n, m}\right|<\frac{1}{m}$ everywhere (cf. [30], Corollary 5.4 and the subsequent comments). If we assume that the "temporal" version of $5^{\bullet}$ holds, then we can write that for any $n, m \in \mathbb{N}$,

$$
\int_{\mathcal{M}} \mathcal{T}_{n, m} d \mu \leq \int_{\mathcal{M}} \mathcal{T}_{n, m} d v
$$

With the aid of Lebesgue's dominant convergence theorem, we can go with $m$ and then with $n$ to infinity, thus obtaining $\int_{\mathcal{M}} f d \mu \leq \int_{\mathcal{M}} f d v$.

Remark 2. In condition $4^{\bullet}$, one can equivalently use the closed half-lines. In other words, $4^{\bullet}$ is equivalent to the following condition:

$4^{\prime \bullet}$ For any time function $\mathcal{T}$ and any $\alpha \in \mathbb{R}$,

$$
\mu\left(\mathcal{T}^{-1}([\alpha,+\infty))\right) \leq v\left(\mathcal{T}^{-1}([\alpha,+\infty))\right) .
$$


Proof. To move between conditions (4) and (11), one can simply use the facts that

$$
\mathcal{T}^{-1}([\alpha,+\infty))=\bigcap_{n=1}^{\infty} \mathcal{T}^{-1}\left(\left(\alpha-\frac{1}{n},+\infty\right)\right) \quad \text { and } \quad \mathcal{T}^{-1}((\alpha,+\infty))=\bigcup_{n=1}^{\infty} \mathcal{T}^{-1}\left(\left[\alpha+\frac{1}{n},+\infty\right)\right)
$$

and employ the continuity of measures from below and above.

\section{Conclusions}

In this work, we have defined the natural extension of the Sorkin-Woolgar relation $\mathrm{K}^{+}$onto $\mathscr{P}(\mathcal{M})$ and studied its basic properties and characterizations. Since $\mathrm{K}^{+}=J^{+}$for causally simple spacetimes, many of the results obtained here reduce to those known from [12]. However, the current paper yields two new properties of the causal precedence relation $\preceq$ on $\mathscr{P}(\mathcal{M})$ for causally simple $\mathcal{M}$. Namely, by Proposition 1 , the relation $\preceq$ on $\mathscr{P}(\mathcal{M})$ is closed, whereas by Theorem 2, conditions $4^{\bullet}$ and $4^{\prime \bullet}$ provide additional characterizations of $\mu \preceq v$ in terms of inverse images of half-lines under time functions. Finally, observe that Remark 1 -making reference neither to $\preceq$ nor $\preceq_{K}$ 一 generalizes ([12], Theorem 6).

Let us remark that all the obtained results would also hold if one, instead of $\mathrm{K}^{+}$, considered another well-studied causal relation introduced by Seifert [31] and often denoted $J_{S}^{+}$. Of course, one would readily define its extension onto $\mathscr{P}(\mathcal{M})$ through the obvious replacements in Definition 2. In fact, even obtaining the proofs of the "Seifert" versions of Proposition 1 and Theorem 2 would simply amount to swapping $\mathrm{K}^{+}$for $J_{S}^{+}$throughout the whole of Section 2 . This is because $J_{S}^{+}$- just like $\mathrm{K}^{+}$-is always transitive and topologically closed; moreover, if the underlying spacetime is stably causal, then actually $J_{S}^{+}=K^{+}[27]$.

There are still some natural questions concerning the extension of $K^{+}$onto $\mathscr{P}(\mathcal{M})$ to be addressed. In future work, we shall investigate, for example, the antisymmetry of $\preceq_{K}$ for measures, which for pointlike events is known to hold exactly in stably causal spacetimes [27]. The question is whether stable causality already guarantees that $\mu \preceq_{K} v \preceq_{K} \mu \Rightarrow \mu=v$, or if rather one has to impose some stronger requirements on the causal properties of $\mathcal{M}$, such as causal continuity. Another idea would be to try to recast the results of [16], which pertain to (the measure-theoretic extension of) $J^{+}$ and to $C^{2}$-metrics, into the more general setting of the Sorkin-Woolgar causality and the metrics of lower regularity.

Acknowledgments: The author wishes to thank Stefan Suhr for his clarifying comments on Theorem 3.

Conflicts of Interest: The author declares no conflict of interest.

\section{Appendix A}

For the reader's convenience, below we outline some topological and measure-theoretic notions and results used in the paper.

Polish spaces are completely metrizable spaces possessing a countable dense subset. Their basic examples include (second countable) manifolds as well as certain spaces of continuous functions ([32], Appendix A). They turn out to have "nice" measure-theoretic properties (exemplified by the two theorems below), which render them extremely useful in probability theory [33] and optimal transport theory $[13,34]$.

The space $\mathscr{P}(\mathcal{Y})$ of all Borel probability measures on a Polish space $\mathcal{Y}$ is usually endowed with the narrow topology, defined as the coarsest topology such that for each $f \in C_{b}(\mathcal{Y})$ the real-valued map $\mathscr{P}(\mathcal{Y}) \ni \mu \mapsto \int_{\mathcal{Y}} f d \mu$ is continuous. Thus, a sequence $\left(\mu_{n}\right) \subseteq \mathscr{P}(\mathcal{Y})$ is narrowly convergent to $\mu \in \mathscr{P}(\mathcal{Y})$ iff $\int_{\mathcal{Y}} f d \mu_{n} \rightarrow \int_{\mathcal{Y}} f d \mu$ for every $f \in C_{b}(\mathcal{Y})$.

It can be shown that $\mathscr{P}(\mathcal{Y})$ is a Polish space as well ([33], Chapter 18, Theorem 24), and hence narrowly convergent sequences fully determine the narrow topology. 
Two seminal results of the probability theory on Polish spaces used in this paper are the Portmanteau theorem and the Prokhorov theorem ([33], Chapter 18, Theorems $6 \& 17$, respectively). The former provides a list of equivalent conditions for a sequence $\left(\mu_{n}\right) \subseteq \mathscr{P}(\mathcal{Y})$ to be narrowly convergent to some $\mu$. In particular, in the paper we make use of the following characterization:

$$
\mu_{n} \rightarrow \mu \text { narrowly } \Leftrightarrow \mu(F) \geq \limsup _{n \rightarrow+\infty} \mu_{n}(F) \quad \text { for any closed } F \subseteq \mathcal{Y} \text {. }
$$

Prokhorov's theorem, on the other hand, characterizes the relatively compact subsets of $\mathscr{P}(\mathcal{Y})$, i.e., the subsets whose closure is compact (in the narrow topology). Concretely, $\mathcal{A} \subseteq \mathscr{P}(\mathcal{Y})$ is relatively compact iff it is tight, that is iff

$$
\forall \varepsilon>0 \quad \exists \text { compact } K_{\varepsilon} \subseteq \mathcal{Y} \quad \forall v \in \mathcal{A} \quad v\left(K_{\varepsilon}\right) \geq 1-\varepsilon
$$

This in particular implies that any Borel probability measure on a Polish space is inner regular, i.e., for any $\mu \in \mathscr{P}(\mathcal{Y})$ and any Borel $\mathcal{X} \subseteq \mathcal{Y}$

$$
\mu(\mathcal{X})=\sup \{\mu(\mathcal{K}) \mid \mathcal{K} \subseteq \mathcal{X}, \mathcal{K}-\text { compact }\}
$$

\section{References}

1. Minguzzi, E.; Sánchez, M. The causal hierarchy of spacetimes. In Recent Developments in Pseudo-Riemannian Geometry (ESI Lectures in Mathematics and Physics); Alekseevsky, D.V., Baum, H., Eds.; European Mathematical Society Publishing House: Scheuchzerstrasse, Switzerland, 2008; pp. 299-358.

2. Beem, J.; Ehrlich, P.; Easley, K. Global Lorentzian Geometry; Monographs and Textbooks in Pure and Applied Mathematics; CRC Press: Boca Raton, FL, USA, 1996.

3. Penrose, R. Techniques of Differential Topology in Relativity; CBMS-NSF Regional Conference Series in Applied Mathematics; SIAM: Philadelphia, PA, USA, 1972.

4. O'Neill, B. Semi-Riemannian Geometry with Applications to Relativity; Academic Press: Cambridge, MA, USA, 1983.

5. Hawking, S.W.; Ellis, G.F.R. The Large Scale Structure of Space-Time; Cambridge Books Online; Cambridge University Press: Cambridge, UK, 1973.

6. Wald, R.M. General Relativity; University of Chicago Press: Chicago, IL, USA, 1984.

7. Franco, N.; Eckstein, M. An algebraic formulation of causality for noncommutative geometry. Class. Quantum Gravity 2013, 30, 135007.

8. Franco, N. Lorentzian Approach to Noncommutative Geometry. arXiv 2011, arXiv:1108.0592.

9. Franco, N.; Eckstein, M. Exploring the causal structures of almost commutative geometries. Symmetry Integr. Geom. Meth. Appl. 2014, 10, 010.

10. Eckstein, M.; Franco, N. Causal structure for noncommutative geometry. In Proceedings of the 2014 Frontiers of Fundamental Physics, Marseille, France, 15-18 July 2014.

11. Franco, N.; Eckstein, M. Causality in noncommutative two-sheeted space-times. J. Geom. Phys. 2015, $96,42-58$,

12. Eckstein, M.; Miller, T. Causality for nonlocal phenomena. arXiv 2015, arXiv:1510.06386.

13. Ambrosio, L.; Gigli, N. A User's Guide to Optimal Transport. In Modelling and Optimisation of Flows on Networks; Piccoli, B., Rascle, M., Eds.; Springer: Berlin/Heidelberg, Germany, 2013.

14. Villani, C. Optimal Transport: Old and New; Volume 338, Grundlehren der Mathematischen Wissenschaften; Springer: Berlin/Heidelberg, Germany, 2008.

15. Suhr, S. Theory of optimal transport for Lorentzian cost functions. arXiv 2016, arXiv:1601.04532.

16. Miller, T. Polish spaces of causal curves. J. Geom. Phys. 2017, 116, 295-315.

17. Eckstein, M.; Miller, T. Causal evolution of wave packets. Phys. Rev. A 2017, 95, 032106.

18. Gerlach, B.; Gromes, D.; Petzold, J.; Rosenthal, P. Über kausales Verhalten nichtlokaler Größen und Teilchenstruktur in der Feldtheorie. Z. Phys. 1968, 208, 381-389.

19. Gerlach, B.; Gromes, D.; Petzold, J. Energie und Kausalität. Z. Phys. 1969, 221, 141-157. 
20. Gromes, D. On the problem of macrocausality in field theory. Z. Phys. 1970, 236, $276-287$.

21. Hegerfeldt, G.C. Remark on causality and particle localization. Phys. Rev. D 1974, 10, 3320-3321.

22. Hegerfeldt, G.C. Violation of causality in relativistic quantum theory? Phys. Rev. Lett. 1985, 54, $2395-2398$.

23. Hegerfeldt, G.C. Causality problems for Fermi's two-atom system. Phys. Rev. Lett. 1994, 72, 596-599.

24. Hegerfeldt, G.C. Particle localization and the notion of Einstein causality. In Extensions of Quantum Theory; Horzela, A., Kapuścik, E., Eds.; Apeiron: Montreal, QC, Canada, 2001; pp. 9-16.

25. Hegerfeldt, G.C.; Ruijsenaars, S.N.M. Remarks on causality, localization, and spreading of wave packets. Phys. Rev. D 1980, 22, 377-384.

26. Sorkin, R.D.; Woolgar, E. A causal order for spacetimes with $C^{0}$ Lorentzian metrics: Proof of compactness of the space of causal curves. Class. Quantum Gravity 1996, 13, 1971-1994.

27. Minguzzi, E. K-Causality Coincides with Stable Causality. Commun. Math. Phys. 2009, 290, 239-248.

28. Minguzzi, E. Time functions as utilities. Commun. Math. Phys. 2010, 298, 855-868,

29. Aliprantis, C.D.; Border, K. Infinite Dimensional Analysis: A Hitchhiker's Guide; Springer: Berlin/Heidelberg, Germany, 2006.

30. Chruściel, P.T.; Grant, J.D.E.; Minguzzi, E. On Differentiability of Volume Time Functions. Annales Henri Poincaré 2016, 17, 2801-2824.

31. Seifert, H.J. The causal boundary of space-times. Gen. Relativ. Gravitat. 1971, 1, 247-259.

32. Kerr, D.; Li, H. Ergodic Theory: Independence and Dichotomies; Springer Monographs in Mathematics; Springer: Berlin/Heidelberg, Germany, 2016.

33. Fristedt, B.E.; Gray, L.F. A Modern Approach to Probability Theory; Probability and Its Applications; Birkhäuser: Basel, Switzerland, 1997.

34. Ambrosio, L.; Gigli, N.; Savaré, G. Gradient Flows: In Metric Spaces and in the Space of Probability Measures; Birkhäuser: Basel, Switzerland, 2008.

(C) 2017 by the author. Licensee MDPI, Basel, Switzerland. This article is an open access article distributed under the terms and conditions of the Creative Commons Attribution (CC BY) license (http:/ / creativecommons.org/licenses/by/4.0/). 